\title{
Seroprevalence of Bartonella infection in American free-ranging and captive pumas (Felis concolor) and bobcats (Lynx rufus)
}

\author{
Bruno B. CHOMEL ${ }^{a *}$, Yoko KIKUCHI ${ }^{a}$, Janice S. MARTENSON ${ }^{b}$, \\ Melodie E. ROELKE-PARKER ${ }^{\mathrm{b}}$, Chao-Chin CHANG $^{\mathrm{a}}$, Rickie W. KASTEN ${ }^{\mathrm{a}}$, \\ Janet E. FOLEY ${ }^{\mathrm{c}}$, John LAUDRE ${ }^{\mathrm{d}}$, Kerry MURPHYe, Pamela K. SWIFT ${ }^{\mathrm{f}}$, \\ Vicki L. KRAMER', Stephen J. O'BRIEN ${ }^{b}$ \\ a Department of Population Health and Reproduction, School of Veterinary Medicine, \\ University of California, Davis, CA 95616, USA \\ b Laboratory of Genomic Diversity, National Cancer Institute, Frederick, MD 21702-1201, USA \\ c Department of Medicine and Epidemiology, School of Veterinary Medicine, University of California, \\ Davis, CA 95616, USA \\ d Idaho State University, Biological Sciences Department, Pocatello, ID PO Box 8007-83209, USA \\ e Hornocker Wildlife Research Institute, PO Box 3246, Moscow, ID 83843-1908, USA \\ ${ }^{\mathrm{f}}$ California Department of Fish and Game, Wildlife Investigation Laboratory, 1701 Nimbus Road, \\ Suite D, Rancho Cordova, CA 95670, USA \\ g California Department of Health Services, Division of Vector borne diseases, 1616 Capitol Ave., \\ MS 7307, Sacramento, CA 94234-7320, USA
}

(Received 5 August 2003; accepted 23 October 2003)

\begin{abstract}
Bartonella henselae is the main agent of cat scratch disease in humans and domestic cats are the main reservoir of this bacterium. We conducted a serosurvey to investigate the role of American wild felids as a potential reservoir of Bartonella species. A total of 479 samples ( 439 serum samples and 40 Nobuto strips) collected between 1984 and 1999 from pumas (Felis concolor) and 91 samples (58 serum samples and 33 Nobuto strips) collected from bobcats (Lynx rufus) in North America, Central America and South America were screened for $B$. henselae antibodies. The overall prevalence of $B$. henselae antibodies was respectively $19.4 \%$ in pumas and $23.1 \%$ in bobcats, with regional variations. In the USA, pumas from the southwestern states were more likely to be seropositive for $B$. henselae (prevalence ratio $(\mathrm{PR})=2.82,95 \%$ confidence interval $(\mathrm{CI})=1.55,5.11$ ) than pumas from the Northwest and Mountain states. Similarly, adults were more likely to be B. henselae seropositive than juveniles and kittens $(\mathrm{PR}=1.77,95 \% \mathrm{CI}=1.07,2.93)$. Adult pumas were more likely to have higher $B$. henselae antibody titers than juveniles and kittens $(p=0.026)$. $B$. henselae antibody prevalence was $22.4 \%(19 / 85)$ in bobcats from the USA and $33.3 \%(2 / 6)$ in the Mexican bobcats. In the USA, antibody prevalence varied depending on the geographical origin of the bobcats. In California, the highest prevalence was in bobcats from the coastal range (37.5\%). These results suggest a potential role of wild felids in the epidemiological cycle of Bartonella henselae or closely related Bartonella species.
\end{abstract}

Bartonella henselae / bobcat / Felis concolor / Lynx rufus / puma

* Corresponding author: bbchomel@ucdavis.edu 


\section{INTRODUCTION}

Bartonella henselae is the main agent of cat scratch disease $[4,7]$. The initial isolation of $B$. henselae from the blood of a cat was reported in 1992 [18]. Since then, a high percentage of domestic cats was found to be bacteremic with $B$. henselae in various parts of the world, with some cats being bacteremic for months $[5,13,15]$. Cats are the main reservoir for $B$. henselae [1, 5, 13], and transmission is mainly from cat to cat by cat fleas (Ctenocephalides felis) [6, $9,11]$. Cats are also the main reservoir for B. clarridgeiae $[10,16]$, a more recently discovered Bartonella species, which may also be one of the etiologic agents of CSD [14]. Finally, B. koehlerae has also been isolated from two cats in California [8] and one French cat [20] and identified by PCR in cat fleas in France [21], but its pathogenic role in CSD is still unknown [8].

Serosurveys for the presence of Bartonella antibodies in domestic cat populations, using an immunofluorescence antibody test, have been conducted in many parts of the world [4], including the United States, where an overall $B$. henselae antibody prevalence of respectively $27.9 \%$ among 628 pet cats from all over North America and $28.2 \%$ of 1314 cats from the USA was reported [3, 12]. In California, $81 \%$ of 205 cats were found positive for $B$. henselae antibody [5]. In the Americas, presence of $B$. henselae antibodies in domestic cats has also been reported from Canada and Chile [12, 17, 25]. In Chile, 54 (71.0\%) of the 76 cats tested were $B$. henselae antibody positive [25].

A sero-epidemiological study of $B$. henselae infection in free-ranging and captive wild felids was conducted a few years ago in California [24]. Fifty-three percent of 62 free-ranging bobcats (Lynx rufus) and $35 \%$ of 74 free-ranging pumas (Felis concolor) trapped in California were seropositive for $B$. henselae, and $30 \%$ of 114 captive wild felids (genera Acinonyx, Panthera and Felis) had B. henselae antibodies. Furthermore, isolation of Bartonella from freeranging pumas and bobcats from northern California has been reported [2]. These Bartonella spp. were experimentally infective for domestic cats [23]. Speciation of these isolates is being presently performed. In Florida panthers (Felis concolor coryi) and pumas (Felis concolor stanleyana) introduced from Texas to Florida for genetic restoration, Rotstein et al. [22] reported a B. henselae antibody prevalence of $18 \%$ $(5 / 28)$ in Florida panthers and of 28\% (2/7) in pumas. Besides these states, no data are available concerning the prevalence of $B$. henselae antibodies among wild felids from other parts of North America as well as Central and South America.

Pumas are currently found in most of South and Central America and in the western part of North America, as far north as southern Alaska, and in Florida. In Florida, pumas, also known as Florida panthers, belong to a subspecies of pumas, which is genetically distinct from other puma subspecies, consistent with a long period of isolation and inbreeding [19]. Bobcats are smaller felids that are quite abundant in North and Central America.

Bartonella henselae is a potentially transmissible infectious agent among domestic cats and wild felids [23]. Furthermore, $B$. henselae is a major zoonotic agent in humans. Therefore, it is important to investigate the presence of this infectious agent in wild cats to determine if wild felids could be a source of infection for domestic animals and humans, and serve as a potential reservoir for this bacterium. The objectives of this study were: (1) to investigate $B$. henselae seropositivity among American pumas and bobcats; and (2) to establish possible risk factors (genus, species, age, and geographical origin) associated with Bartonella seropositivity in these wild felids. 


\section{MATERIALS AND METHODS}

\subsection{Animals and serum samples}

Serum samples or Nobuto filter strips (Advantec/MSS Inc., Pleasanton, California, USA) from 479 free-ranging and captive pumas and 91 free-ranging and captive bobcats were obtained from eight different sources, listed as follows: (1) the National Institutes of Health, Laboratory of Genomic Diversity, National Cancer Institute, Frederick, MD, USA; (2) the California Department of Fish and Game, Rancho Cordova, CA, USA; (3) University of California Davis, School of Veterinary Medicine, Wildlife Health Center, Davis, CA, USA; (4) the Hornocker Wildlife Research Institute, Moscow, ID, USA; (5) the Safari Game Search Foundation Inc., Wiston, OR, USA; (6) the Center for Disease Control and Prevention (CDC), National Center for Infectious Diseases, Division of VectorBorne Infectious Diseases, Fort Collins, CO, USA; and (7) the Idaho State University, Biological Sciences Department, Pocatello, ID, USA. Samples were collected from 1984 through 1999 and serum samples stored at $-20{ }^{\circ} \mathrm{C}$ until tested. There were 439 serum samples and 40 Nobuto strips from pumas, and 58 serum samples and 33 Nobuto strips from bobcats. Seventyfive percent of the serum samples (361/479) and all forty Nobuto strips collected from pumas were from the United States. A total of 137 pumas (97 serum samples and 40 Nobuto strips) and 48 bobcats (15 serum samples and 33 Nobuto strips) were from California. The 73 Nobuto strips used in this study were from pumas or bobcats trapped in California for the plague surveillance program. Country of origin, county of origin from California, estimated age, and sex were available for most of these animals.

The serum and nobuto samples from pumas were collected in 15 different countries: Argentina $(n=25)$, Belize $(n=1)$, Bolivia $(n=5)$, Brazil (States of Sao Paulo, Goias and Tocantinas) $(n=11)$, Canada
(British Columbia) $(n=23)$, Chile $(n=10)$, Costa Rica $(n=5)$, Guatemala $(n=4)$, Mexico $(n=12)$, Nicaragua $(n=5)$, Panama $(n=$ $3)$, Paraguay $(n=3)$, Peru $(n=5)$, USA $(n=$ $361)$ and Venezuela $(n=6)$. Within the USA, pumas were trapped in ten different states: Arizona $(n=15)$, California $(n=$ 137), Colorado $(n=4)$, Florida $(n=37)$, Idaho $(n=57)$, New Mexico $(n=7)$, Oregon $(n=11)$, Texas $(n=40)$, Utah $(n=2)$, and Wyoming $(n=51)$ (Tab. I). The bobcats were trapped in the USA $(n=85)$ and Mexico $(n=6)$. Within the USA, bobcats were trapped in four different states, including California $(n=48)$, Florida $(n=28)$, Nevada $(n=6)$ and Oregon $(n=3)$. All puma and bobcat serum samples from Central (including Mexico) and South Americas, with the exception of three puma serum samples from Chile, were from captive animals.

\subsection{Bartonella antibody testing}

Antibody titers against $B$. henselae were determined using an indirect immunofluorescence antibody (IFA) as previously described [1, 5]. Bartonella henselae (Strain U4; University of California, Davis, USA), originally isolated from a naturally infected cat, was cultivated with Felis catus whole fetus (FCWF) cells in complete cell media for 3 to 5 days for antigen preparation. Forty microliters of antigen solution was applied into each well of twelve well polytetrafluoroethylene-coated slides (Cel-Line Associates, Newfield, NJ, USA), and these slides were incubated for $24 \mathrm{~h}$ to allow the cells to adhere to the slides. Slides were then rinsed with phosphate-buffered saline (PBS; Sigma Diagnostic St. Louis, MO, USA, pH 7.4), air-dried, acetone fixed, and stored at $-20^{\circ} \mathrm{C}$ until they were used. Serum samples were diluted in PBS at 1:32 dilution, and any sample with a titer superior or equal to 1:64 (positive cut-off) sample was run serially at a two-fold dilution to the end point titer. Positive control (cats infected with Strain U4; University of California, Davis, USA) and negative control 
Table I. Prevalence of Bartonella henselae antibodies in free-ranging and captive pumas and freeranging bobcats in different countries from the Americas.

\begin{tabular}{lcc}
\hline Geographic region & $\begin{array}{c}\text { Pumas } \\
\text { Number of positive/ } \\
\text { Number samples tested (\%) }\end{array}$ & $\begin{array}{c}\text { Bobcats } \\
\text { Number of positive/ } \\
\text { Number samples tested (\%) }\end{array}$ \\
\hline USA & $73 / 361(20.2)$ & $19 / 85(22.4)$ \\
Canada & $0 / 23(0.0)$ & N. A. \\
Mexico & $1 / 12(8.3)$ & $2 / 6(33.3)$ \\
Central America and Venezuela & $8 / 24(33.3)$ & N. A. \\
Southern part of South Americab & $11 / 49(22.4)$ & N. A. \\
Andean countries & & N. A. \\
Total & $0 / 10(0.0)$ & $\mathbf{2 1 / 9 1}(\mathbf{2 3 . 1})$ \\
\hline
\end{tabular}

a Central America includes the five following countries: Belize, Guatemala, Panama, Costa-Rica and Nicaragua.

${ }^{b}$ Southern Part of South America includes the four following countries: Argentina, Brazil, Paraguay and Chile.

${ }^{c}$ Andean Countries includes the following two countries: Bolivia and Peru.

N. A.: Not applicable.

serum (SPF cats) were used for each IFA slide. The slides were incubated for $25 \mathrm{~min}$ at $37^{\circ} \mathrm{C}$ and were washed for 5 min twice with PBS. Fluorescein-conjugated goat anti-cat immunoglobulin (Whole-molecule immunoglobulin G; Cappel, Oreganon Teknika Corp., Durham, NC, USA) was diluted to $1: 800$ in PBS with $0.001 \%$ of Evan's blue. Thirty microliters of this conjugate was applied to each well, and slides were incubated for $30 \mathrm{~min}$ at $37{ }^{\circ} \mathrm{C}$ and washed with PBS for 5 min twice. Using a fluorescence microscope (magnification, $\times 400$ ), the intensity of bacillus-specific fluorescence was scored subjectively from 1 to 4 . Fluorescence score of $\geq 2$ at dilution 1:64 was considered to be a positive result. The same two persons read all the IFA slides independently and the endpoint titer was the highest dilution found with a score $\geq 2$ by both readers. Testing was performed only against a $B$. henselae antigen, as serological testing of several puma and bobcat serum samples by IFA with a puma strain gave similar antibody titers when compared to B. henselae IFA slides (Chomel, unpublished data).

Nobuto filter strips, which had been dipped in the animal blood and air dried, were cut thin into two equal pieces into a $1.5 \mathrm{~mL}$ Eppendorf vial and $0.4 \mathrm{~mL}$ of borate buffer (1.5 M NaCl, 0.5 M H3BO3, $1 \mathrm{M} \mathrm{NaOH}$ ), pH 8.0 added. The vial was kept overnight at $4{ }^{\circ} \mathrm{C}$ and then heat-inactivated for $20 \mathrm{~min}$ at $60^{\circ} \mathrm{C}$. The paper strips were pressed with a wood rod and removed then two drops of washed sheep blood cells were added. These vials were left over night at $4{ }^{\circ} \mathrm{C}$. The vials were centrifuged for $5 \mathrm{~min}$ at $2000 \mathrm{rpm}$. These vials were kept frozen at $-20{ }^{\circ} \mathrm{C}$ until tested. The supernatant was used for IFA serology testing. We have previously demonstrated that a dilution at 1:8 of Nobuto extracts was equivalent to a 1:64 dilution for serum samples [24].

Data were entered in an Excel spreadsheet and analyzed using SAS 6.12 and Epi Info $6.04 \mathrm{~b}$ softwares. Prevalence differences were analyzed using Chi-square tests 
Table II. Distribution of Bartonella henselae antibody titers among pumas from the Americas by age group and percent positive $(\geq 64)$ by age group.

\begin{tabular}{lrrrrrrrc}
\hline & \multicolumn{7}{c}{$B$. henselae antibody titers } \\
\cline { 2 - 8 } Age group & 0 & 32 & 64 & 128 & 256 & 512 & 1024 & Total (\%) \\
\cline { 2 - 8 } & \multicolumn{8}{c}{ Number of pumas } \\
\hline Adult & 212 & 2 & 43 & 11 & 8 & 2 & 3 & $67 / 281(23.8)$ \\
Juvenile & 84 & 1 & 6 & 3 & 4 & 0 & 2 & $15 / 100(15)$ \\
Kitten & 18 & 0 & 1 & 0 & 0 & 0 & 0 & $1 / 19(5.3)$ \\
Unknown & 69 & 0 & 8 & 0 & 2 & 0 & 0 & $10 / 79(12.7)$ \\
Total & 383 & 3 & 58 & 14 & 14 & 2 & 5 & $93 / 479(19.4)$ \\
\hline
\end{tabular}

for two by two or $r$ by $k$ tables. For comparison of antibody titers and age in pumas, adults and non-adults (juveniles and kittens) were compared for three variables: negative (titer < 64), positive (titers of 64 to 128 for $B$. henselae) and strong positive ( $\geq 256$ for $B$. henselae).

\section{RESULTS}

A total of 479 puma samples ( 439 serum and 40 Nobuto strips) collected from North America, Central America and South America were screened for antibodies for Bartonella henselae. The age distribution of the animals ranged from 1 month to $>16$ years. Among the pumas for which an estimated age was available $(83.5 \%, 400 /$ 479), the majority $(70.2 \%)$ were adults ( $\geq 24$ months), 25\% ( $n=100)$ were juveniles (6 months to less than 24 months) and $4.8 \%$ of them $(n=19)$ were kittens (<6 months). Fifty-two percent (244/469) of the pumas and 59 percent $(50 / 85)$ of the bobcats were males (sex data were missing for 10 pumas and 6 bobcats). The overall prevalence of Bartonella henselae antibodies in pumas was $19.4 \%$, and ranged from 0\% in Canada, Bolivia and Peru to $33.3 \%$ in Central America and Venezuela (Tab. I). Antibody titers of positive animals ranged from 64 to 1024 , most (78.3\%) of the positive pumas having low titers (64 or 128)
(Tab. II). There was no statistical difference between the prevalence of $B$. henselae antibodies in the various geographical regions (North, Central and South) of the Americas.

Among the 361 puma samples (321 serum samples and 40 Nobuto strips) collected in the United States and tested for B. henselae, $73(20.2 \%)$ were seropositive. Antibody prevalence for $B$. henselae varied widely among the various geographic locations where the samples were collected (Tab. III). More specifically, pumas from the Southwestern states (Arizona, California, and New Mexico) were almost three times more likely to be seropositive for $B$. henselae than pumas from the Northwestern and Mountain states (Colorado, Idaho, Oregon, Utah, Wyoming) (Prevalence ratio $(\mathrm{PR})=2.82,95 \%$ Confidence Interval $(\mathrm{CI})=1.55,5.11)$.

In California, antibodies to B. henselae were found in $28.5 \%$ of the 137 pumas tested (Tab. IV). In that state, young pumas (juveniles and kittens) had higher antibody prevalence $(36.7 \%, 11 / 30)$ than adults $(25.2 \%, 25 / 99)$. In Florida, none of the 18 juvenile panthers had Bartonella antibodies. Prevalence of $B$. henselae antibodies in pumas was not statistically different among the various geographic regions of California (Tab. IV).

Overall, antibody prevalence for $B$. henselae increased with age in pumas (Tab. II). 
Table III. Prevalence of Bartonella henselae antibodies in free-ranging pumas and bobcats from different states of the USA.

\begin{tabular}{lcc}
\hline States & $\begin{array}{c}\text { Pumas } \\
\text { P/N }(\%)^{\mathrm{a}}\end{array}$ & $\begin{array}{c}\text { Bobcats } \\
\text { P/N }(\%)\end{array}$ \\
\hline Arizona & $4 / 15(26.7)$ & N. A. \\
California & $39 / 137(28.5)$ & $14 / 48(29.2)$ \\
Colorado & $0 / 4(0.0)$ & N. A. \\
Florida & $2 / 37(5.4)$ & $3 / 28(10.7)$ \\
Idaho & $5 / 57(8.8)$ & N. A. \\
Nevada & N. A. & $1 / 6(16.7)$ \\
New Mexico & $0 / 7(0.0)$ & N. A. \\
Oregon & $1 / 11(9.1)$ & $1 / 3(33.3)$ \\
Texas & $16 / 40(40.0)$ & N. A. \\
Utah & $0 / 2(0.0)$ & N. A. \\
Wyoming & $6 / 51(11.8)$ & N. A. \\
Total & $73 / 361(20.2)$ & $19 / 85(22.4)$ \\
\hline
\end{tabular}

a Number of positive samples/number of samples tested (percent).

b N. A.: Not applicable.
Sixty-seven (23.8\%) of the 281 adults were seropositive for $B$. henselae, whereas only $15 \%$ of the 100 juveniles and $5.3 \%$ of the 19 kittens were seropositive. Adult pumas were 1.77 times $(95 \% \mathrm{CI}=1.07,2.93)$ more likely to have $B$. henselae antibodies than juveniles and kittens. Adult pumas were also more likely to have higher $B$. henselae antibody titers than juveniles and kittens $(p=0.026)$. Similar associations were identified for pumas from the USA, where adults were two times more likely to be $B$. henselae seropositive than juveniles and kittens $(\mathrm{PR}=2.06,95 \% \mathrm{CI}=1.15,3.68)$. They were also more likely to have higher $B$. henselae antibody titers $(p=0.005)$. None of the juveniles from Texas $(0 / 4)$ or Florida (0/18) had Bartonella antibodies, whereas $44.4 \%(16 / 36)$ and $11.1 \%(2 / 18)$ of the adults were seropositive. There were no prevalence differences by sex for $B$. henselae antibodies, as $20.9 \%(51 / 244)$ of the males and $17.3 \%(39 / 225)$ of the females were seropositive.

A total of 91 bobcat samples (58 serum samples and 33 Nobuto strips) were tested

Table IV. Prevalence of Bartonella henselae antibodies in free-ranging pumas and bobcats from different regions of California.

\begin{tabular}{lcc}
\hline Geographic region & Pumas & Bobcats \\
& P/N $(\%)^{\mathrm{a}}$ & P/N (\%) \\
\hline Coastal Range $^{\mathrm{b}}$ & $10 / 61(16.4)$ & $12 / 32(37.5)$ \\
Sierra Nevada $^{\mathrm{c}}$ & $17 / 52(32.7)$ & $1 / 9(11.1)$ \\
Central Valley $^{\mathrm{d}}$ & $0 / 5(0.0)$ & $0 / 3(0)$ \\
Southern California & $10 / 14(71.4)$ & $1 / 4(25)$ \\
Unknown $^{\mathrm{e}}$ & $2 / 5(0.4)$ & $\mathrm{N} . \mathrm{A}$. \\
\hline Total & $39 / 137(28.5)$ & $14 / 48(29.2)$ \\
\hline
\end{tabular}

a Number of positive samples/number of samples tested (percent).

b Coastal Range includes the 18 following counties: Alameda, Contra Costa, Del Norte, Humboldt, Lake, Marin, Mendocino, Monterey, Napa, San Benito, San Luis Obispo, San Mateo, Santa Barbara, Santa Clara, Santa Cruz, Siskiyou, Sonoma, Trinity.

${ }^{\mathrm{c}}$ Sierra Nevada includes the 16 following counties: Amador, Butte, Calaveras, El Dorado, Inyo, Lassen, Mariposa, Modoc, Mono, Nevada, Placer, Plumas, Shasta, Sierra, Tuolumne, Yuba.

d Central Valley includes the 15 following counties: Colusa, Fresno, Glenn, Kings, Kern, Sacramento, San Joaquin, Solano, Stanislaus, Sutter, Madera, Merced, Tehama, Tulare, Yolo.

e Southern California includes the 7 following counties: Imperial, Los Angeles, Orange, Riverside, San Bernardino, San Diego, Ventura. 
for B. henselae antibodies. All but six samples (from Mexico) were from the USA. Bartonella henselae antibody prevalence was $22.4 \%(19 / 85)$ in bobcats from the USA and 33.3\% (2/6) in the Mexican bobcats (Tab. I). In the USA, antibody prevalence varied from $10.7 \%$ in Florida to $33.3 \%$ in Oregon, depending on the geographical origin of the bobcats (Tab. III). In California, the highest prevalence was in bobcats from the coastal range $(37.5 \%)$ whereas none of the three bobcats captured in the central valley region were seropositive (Tab. IV). In Santa Clara County, 9 $(56 \%)$ of 16 bobcats were seropositive, whereas none of the 7 bobcats from Mendocino County had Bartonella antibodies. The only bobcat from Marin County was seropositive. No sex differences in $B$. henselae antibody prevalence were observed between males $(22 \%, 11 / 50)$ and females (26\%, 9/35). Among the 60 bobcats for which an age was given, $92 \%$ (55/60) were adults. Antibody prevalence was $32.7 \%$ $(18 / 55)$ in adult bobcats, whereas none of the five juveniles had B. henselae antibody.

\section{DISCUSSION}

This study confirms the previous reports of Bartonella antibodies in Californian pumas and bobcats [24] and Florida panthers [22], and extends the known distribution of this infection throughout most of the geographical range of these wild felids. It is the first report of Bartonella antibodies in free-ranging and captive pumas and bobcats from various parts of North, Central and South America. Antibody prevalence varied depending upon the origin of the animals within their natural geographic range. Overall, there was no statistical difference between the prevalence of $B$. henselae antibodies in pumas from the three main geographical regions (North, Central, and South) of the Americas.

Within the United States, overall prevalence $(20.2 \%)$ of $B$. henselae antibodies from free-ranging pumas was close to the prevalence previously reported in domestic cats $(27 \%$ to $28 \%)$ [3, 12]. With the exception of Florida, where the puma population is endangered, wild cats from the warm, southern states (such as Arizona, California and Texas) had a higher seroprevalence than animals from the mountainous or more northern states (such as Colorado, Idaho, Utah, Wyoming and Oregon). This distribution superposes well with the reported distribution of Bartonella infection in domestic cats [12], and also fits with the known distribution of the main vectors of Bartonella spp., especially fleas, as reported for domestic cats $[6,12,13]$. In free-ranging pumas from California, we found a seroprevalence of $28.5 \%$, which is close to the $35 \%$ previously reported for 74 free-ranging California pumas [24]. We found a higher Bartonella antibody prevalence in pumas from Texas $(40 \%, 16 / 40)$ than the prevalence of $28 \%(2 / 7)$ reported by Rotstein et al. [22]. Such a difference is likely related to a random sampling error, despite a slightly larger sample size in our study. Similarly, the time of collection of samples varied between studies and may have led to differences in prevalence of infection. On the contrary, we found a much lower prevalence $(5.4 \%, 2 / 37)$ in Florida panthers than the $18 \%(5 / 28)$ reported by Rotstein et al. [22], especially when comparing juvenile pumas. In our study, none of the 18 juveniles tested had Bartonella antibodies, whereas $40 \%$ (4/10) were positive in Rotstein et al.'s study [22]. Continuing surveillance of Bartonella antibodies in Florida panthers will be necessary to determine if infection is spreading among these animals. The Bartonella antibody prevalence for California bobcats in our study was somehow lower $(29.2 \%, 14 / 48)$ than previously reported $(53 \%, 33 / 62)$ [24]. This difference may be attributable to the geographical origin of our respective bobcat populations. The majority of our bobcat samples were collected from the Central and South coast of California (24/48), whereas in the previous study, $39 \%$ of the bobcat samples were collected from the 
North coast of California (24/62). Interestingly, none of the seven bobcats from Mendocino County in this study and none of the seven bobcats from the same county in the previous study were seropositive [24]. Similarly, seroprevalence was almost identical for bobcats from Santa Clara County $(56 \%$ $(9 / 16)$ in this study compared to 53\% (10/ 19) in the previous study).

Overall, B. henselae antibody prevalence in pumas increased with age, which can be explained by a regular exposure to the infectious agent with time. This observation was also supported by higher antibody titers in adults than in young (juveniles and kittens) pumas. Only one kitten was seropositive (titer of 64), and only two juvenile pumas had antibody titers $>256$ versus five adults. However, in California, a higher prevalence in young freeranging pumas was observed, as previously reported [24]. The antibody prevalence in young pumas was also very similar $(36.7 \%$ compared to $40 \%$ ) in both studies. Such a difference for California warrants further investigation. As previously reported [22, 24], no difference in Bartonella antibody prevalence by gender was observed.

The results of the present study indicate the presence of Bartonella antibodies in wild felids in the Americas. It will be important to determine if such felids are reservoirs of Bartonella species that are restricted to their own species. Further studies will be necessary to determine the dynamics of Bartonella infection within the puma and bobcat populations and the possible transmission of Bartonella spp. from wild felids to domestic cats or from the domestic reservoir to wildlife. Similarly, it will be important to determine if the vectors involved in the transmission of Bartonella in wild felids are similar or different from the ones involved in the Bartonella transmission among domestic cats.

\section{ACKNOWLEDGMENTS}

The authors would like to thank H. Ernest (Wildlife Health Center, School of Veterinary
Medicine, U.C. Davis), B.C. Barr (California Veterinary Diagnosis Laboratory System, U.C. Davis), J.E. Bracher (Centers for Disease Control and Prevention (CDC), Division of Vector-Borne Infectious Diseases, Fort Collins, Colorado), S.K. Taylor, (Florida Game and Fish, Naples, Florida), K. Jones (California Department of Fish and Game, Wildlife Investigation Laboratory, Rancho Cordova, California), C. Serfling (Safari Game Search Foundation Inc., American Association of Zoological Parks and Aquariums, Winston, Oregon), V.C. Bleich and B. Pierce (CDFG, Bishop, California) for providing samples and A. Poland (Center for Companion Animal Health, SVM, U.C. Davis, California) for her help in making the IFA slides. Dr. Kikuchi was the recipient of the 1998-1999 Harley Walker research scholarship, School of Veterinary Medicine, University of California, Davis.

\section{REFERENCES}

[1] Abbott R.C., Chomel B.B., Kasten R.W., Floyd-Hawkins K.A., Kikuchi Y., Koehler J.E., Pedersen N.C., Experimental and natural infection with Bartonella henselae in domestic cats, Comp. Immunol. Microbiol. Infect. Dis. 20 (1997) 41-51.

[2] Breischwerdt E.B., Kordick D.L., Bartonella infection in animals: carriership, reservoir potential, pathogenicity, and zoonotic potential for human infection, Clin. Microbiol. Rev. 13 (2000) 428-438.

[3] Childs J.E., Olson J.G., Wolf A., Cohen N., Fakile Y., Rooney J.A., Bacellar F., Regnery R.L., Prevalence of antibodies to Rochalimaea species (cat-scratch disease agent) in cats, Vet. Rec. 136 (1995) 519-520.

[4] Chomel B.B., Cat Scratch Disease, Rev. Sci. Tech. 19 (2000) 136-150.

[5] Chomel B.B., Abbott R.C., Kasten R.W., Floyd-Hawkins K.A., Kass P.H., Glaser C.A., Pedersen N.C., Koehler J.E., Bartonella henselae prevalence in domestic cats in California: risk factors and association between bacteremia and antibody titers, J. Clin. Microbiol. 33 (1995) 2445-2450.

[6] Chomel B.B., Kasten R.W., Floyd-Hawkins K.A., Chi B., Yamamoto K., Roberts-Wilson J., Gurfield A.N., Abbott R.C., Pedersen N.C., Koehler J.E., Experimental transmission of Bartonella henselae by the cat flea, J. Clin. Microbiol. 34 (1996) 1952-1956. 
[7] Dolan M.J., Wong M.T., Regnery R.L., Jorgensen J.H., Garcia M., Peters J., Drehner D., Syndrome of Rochalimaea henselae adenitis suggesting cat scratch disease, Ann. Intern. Med. 118 (1993) 331-336.

[8] Droz S., Chi B., Horn E., Steigerwalt A.G., Whitney A.M., Brenner D.J., Bartonella koehlerae sp. nov., isolated from cats, J. Clin. Microbiol. 37 (1999) 1117-1122.

[9] Foil L., Andress E., Freeland R.L., Roy A.F., Rutledge R., Triche P.C., O'Reilly K.L., Experimental infection of domestic cats with Bartonella henselae by inoculation of Ctenocephalides felis (Siphonaptera: Pulicidae) feces, J. Med. Entomol. 35 (1998) 625-628.

[10] Gurfield A.N., Boulouis H.J., Chomel B.B., Kasten R.W., Heller R., Bouillin C., Gandoin C., Thibault D., Chang C.C., Barrat F., Piemont Y., Epidemiology of Bartonella infection in domestic cats in France, Vet. Microbiol. 80 (2001) 185-198.

[11] Higgins J.A., Radulovic S., Jaworski D.C., Azad A.F., Acquisition of the cat Scratch disease agent Bartonella henselae by cat fleas (Siphonaptera: Pulicidae), J. Med. Entomol. 33 (1996) 490-495.

[12] Jameson P., Greene C., Regnery R., Dryden M., Marks A., Brown J., Cooper J., Glaus B., Greene R., Prevalence of Bartonella henselae antibodies in pet cats through regions of North America, J. Infect. Dis. 172 (1995) 1145-1149.

[13] Koehler J.E., Glaser C.A., Tappero J.W., Rochalimaea henselae Infection. A new zoonosis with the domestic cat as reservoir, JAMA 271 (1994) 531-535.

[14] Kordick D.L., Hilyard E.J., Hadfield T.L., Wilson K.H., Steigerwalt A.G., Brenner D.J., Breitschwerdt E.B., Bartonella clarridgeiae, a newly recognized zoonotic pathogen causing inoculation papules, fever, and lymphadenopathy (cat scratch disease), J. Clin. Microbiol. 35 (1997) 1813-1818.

[15] Kordick D.L., Wilson K.H., Sexton D.J., Hadfield T.L., Berkhoff H.A., Breitschwerdt E.B., Prolonged Bartonella bacteremia in cats associated with cat scratch disease patients, J. Clin. Microbiol. 33 (1995) 32453251 .

[16] Lawson P.A., Collins M.D., Description of Bartonella clarridgeiae sp. nov. isolated from the cat of a patient with Bartonella henselae septicemia, Med. Microbiol. Lett. 5 (1996) 64-73.

[17] Leighton F.A., Artsob H.A., Chu M.C., Olson J.G., A serological survey of rural dogs and cats on the southwestern Canadian prairie for zoonotic pathogens, Can. J. Public Health 92 (2001) 67-71.

[18] Regnery R.L., Martin M., Olson J.G., Naturally occurring Rochalimaea henselae infection in domestic cat, Lancet 340 (1992) $557-$ 558.

[19] Roelke M.E., Martenson J.S., O’Brien S.J., The consequences of demographic reduction and genetic depletion in the Florida panther, Curr. Biol. 3 (1993) 340-350.

[20] Rolain J.M., Fournier P.E., Raoult D., Bonerandi J.J., First isolation and detection by immunofluorescence assay of Bartonella koehlerae in erythrocytes from a French cat, J. Clin. Microbiol. 41 (2003) 4001-4002.

[21] Rolain J.M., Franc M., Davoust B., Raoult D., Molecular detection of Bartonella quintana, B. koehlerae, B. henselae, B. clarridgeiae, Rickettsia felis, and Wolbachia pipientis in cat fleas, France, Emerg. Infect. Dis. 9 (2003) 338-342.

[22] Rotstein D.S., Taylor S.K., Bradley J., Breitschwerdt E.B., Prevalence of Bartonella henselae antibody in Florida panthers, J. Wildl. Dis. 36 (2000) 157-160.

[23] Yamamoto K., Chomel B.B., Kasten R.W., Chang C.C., Tseggai T., Decker P.R., Mackowiak M., Floyd-Hawkins K.A., Pedersen N.C., Homologous protection but lack of heterologous protection by various species and types of Bartonella in specific pathogen free cats, Vet. Immunol. Immunopathol. 65 (1998) 191-204.

[24] Yamamoto K., Chomel B.B., Lowenstine L.J., Kikuchi Y., Phillips L.G., Barr B.C., Swift P.K., Jones K.R., Riley S.P., Kasten R.W., Foley J.E., Pedersen N.C., Bartonella henselae antibody prevalence in free-ranging and captive wild felids from California, J. Wildl. Dis. 34 (1998) 56-63.

[25] Zaror L., Ernst S., Navarrete M., Ballesteros A., Boroscheck D., Ferres M., Thibaut J., Serologic detection of Bartonella henselae in cats in the city of Valdivia, Chile, Arch. Med. Vet. 34 (2002) 103-110. 